\title{
Impact of bowel preparation on caecal intubation time during colonoscopy
}

\author{
Haris Alvi ${ }^{1}$, Tazeen Rasheed ${ }^{2}$, Majid Ahmed Shaikh ${ }^{3}$, \\ Faiza Sadaqat Ali ${ }^{4}$, Baber Faiyaz Zuberi ${ }^{5}$, Asad Ali Samejo ${ }^{6}$
}

\begin{abstract}
Objective: To determine the caecal intubation time depending on bowel preparation as per Boston Bowel Preparation Scale.

Methods: This cross-sectional study was conducted at Dr. Ruth K. M. Pfau, Civil Hospital Karachi between August 2018 to February 2019. A total of 201 patients were included in the study. Time was recorded from insertion of colonoscope to the time required to reach the cecum. Bowel preparation was graded during withdrawal of colonoscope by using Boston Bowel Preparation Scale. Pearson Correlation test was used to study correlation of BBPS scores with CIT, gender, BMI, adenoma and polyp detection.

Results: In this study 201 patients undergoing colonoscopy were included. Mean \pm SD of age of patients was $36.9 \pm 15.8$ years. Out of the 201 patients $112(56 \%)$ were males and $89(44 \%)$ were females. The results of our study showed that increased Boston Bowel Preparation Scale Scores were associated with decreased caecal intubation time. The mean CIT was $10.7 \pm 5.4$ minutes and Pearson correlation was significant at 0.002. Significant correlations of BBPS were also found with BMI and adenoma detection.
\end{abstract}

Conclusion: The diagnostic effectiveness of colonoscopy depends upon the quality of the preparation. Good bowel preparation improves the speed of colonoscopy and its completeness.

KEYWORDS: Boston bowel preparation scale; Colonoscopy; Caecal intubation time; Adenoma Detection Rate.

How to cite this:

doi: https://doi.org/10.12669/pjms.35.6.1031

Alvi H, Rasheed T, Shaikh MA, Ali FS, Zuberi BF, Samejo AA. Impact of bowel preparation on caecal intubation time during colonoscopy. Pak J Med Sci. 2019;35(6):1516-1519. doi: https://doi.org/10.12669/pjms.35.6.1031

This is an Open Access article distributed under the terms of the Creative Commons Attribution License (http://creativecommons.org/licenses/by/3.0), which permits unrestricted use, distribution, and reproduction in any medium, provided the original work is properly cited.

1. Prof. Haris Alvi, MBBS, FCPS.

2. Dr. Tazeen Rasheed

Assistant Professor,

3. Dr. Majid Ahmed Shaikh

Assistant Professor,

4. $\quad$ Dr. Faiza Sadaqat Ali

Senior Registrar,

5. Prof. Bader Faiyaz Zuberi

6. Dr. Asad Ali Samejo Postgraduate Trainee,

1-6: Dow University of Health Sciences,

Karachi, Pakistan.

Correspondence:

Prof. Bader Faiyaz Zuberi,

C-404, Al-Habib Pride,

CL-8/5, Civil Lines,

Karachi, Pakistan.

Email: bader@zuberi.net

* Received for Publication:

* Revision Received:

* Revision Accepted:
May 10, 2019

August 24, 2019

August 26, 2019

\section{INTRODUCTION}

Caecal intubation time is defined as the time required to reach caecum after insertion of colonoscope. It indicates the difficulty of advancing the scope till caecum. ${ }^{1}$ It is affected by many factors including age, sex, increased or decreased body mass index, waist circumference, history of abdominal or pelvic surgery, experience of endoscopist, and the bowel preparation. ${ }^{1-3}$ It is also affected by length of colonoscope and advance features present in colonoscope such as variable stiffness. ${ }^{4}$ Although other factors are measurable and obtained from history, but bowel preparation is measured by different scales including American Society for Gastrointestinal Endoscopy (ASGE) and American Gastroenterological Association (AGA) Task Force who used the terms as "excellent, good, fair and 
poor". This lacks standard definition and results in variability in comments on bowel preparation.., 6

Another scale named as Boston Bowel Preparation Scale (BBPS) is considered as a novel scale for rating of bowel cleanliness has received good intra and inter-observer reliability assessments and is designed for researches related to colonoscopy and bowel preparation. ${ }^{78}$ It assesses the bowel during withdrawal after washing and suctioning. ${ }^{8}$ BBPS scores correlates with polyp detection rates and caecal intubation time. ${ }^{5,8}$ Lower BBPS score is associated with missed polyp detection and requires early repeat colonoscopy as it has negative impact on colon cancer prevention. ${ }^{6,9}$

Our objective was to determine the impact of bowel preparation on caecal intubation time, as good bowel preparation will lead to shorter caecal intubation time, better visualization of mucosa and polyp detection.

\section{METHODS}

This cross-sectional study was conducted at Dr. Ruth K. M. Pfau, Civil Hospital Karachi between August 2018 to February 2019. Non-probability consecutive sampling was used for selection of patients. Approval was taken from the Institutional Review Board of Dow University of Health Sciences. Inclusion criteria: All patients between the ages of 18-70 years undergoing colonoscopy were included. Exclusion criteria: Patients who were unable to tolerate colonoscopy preparation solutions and patients having electrolyte abnormalities to whom colonoscopy preparation solution could not be given, were excluded.

A written informed consent was taken from the patients undergoing colonoscopy. Patients were advised to take colonoscopy preparation solution at $12 \mathrm{PM}$ then at $6 \mathrm{PM}$ the day before colonoscopy. They were advised to take liquid diet for 24 hours before colonoscopy and nil oral orders for four hours before colonoscopy. Each colonoscopy preparation kit contains two $45 \mathrm{ml}$ bottles of solution. Each $45 \mathrm{ml}$ bottle contains: sodium sulfate 17.5 grams, potassium sulfate 3.13 grams, magnesium sulfate 1.6 grams. Inactive ingredients include sodium benzoate NF, sucralose, malic acid FCC, citric acid USP, flavoring ingredients, purified water, USP. The solution is diluted to a final volume of $500 \mathrm{ml}$ with water before intake. Colonoscopy were done by three skilled colonoscopists which in study were called as A, B \& C. Time was recorded from insertion of colonoscope in anus till caecum is reached. Bowel preparation was graded during withdrawal of colonoscope by using BBPS from 0 to 3, where higher score means good bowel preparation, details of Boston Bowel Scale is given as under:

$0=$ poor preparation with solid stool on mucosa that cannot be cleaned and hinder the mucosal visualization.

1 = some mucosa seen while rest of mucosa cannot be seen due to solid or loose stool.

2 = some mucosa cannot be seen due to solid or loose stool while rest of mucosa is visualized.

$3=$ good preparation and visualization of entire mucosa. ${ }^{7}$

It is the sum of three individual segments scale.

First

Segment

Caecum \& Ascending Colon

Second Transverse Colon with Hepatic \&

Segment Splenic Flexures

Third Descending, Sigmoid Colon \&

Segment Rectum

The impact of bowel preparation on caecal intubation time was analyzed separately for individual colonoscopists as well as combined for all three too. BBPS scores was correlated with CIT, gender, BMI, adenoma and polyp detection using 'Pearson Correlation Test'. Value of $\leq 0.05$ was taken as significant.

\section{RESULTS}

In this study 201 patients undergoing colonoscopy were included. Three colonoscopists from our department participated in this study. Out of the 201 colonoscopies, 72 were performed by Colonoscopist-A, 35 by Colonoscopist-B and 94 by Colonoscopist-C. Mean \pm SD of age of patients was $36.9 \pm 15.8$ years. Out of the 201 patients 112 $(56 \%)$ were males and $89(44 \%)$ were females. Mean age of males was $34.8 \pm 16.6$ years while that of females was $39.6 \pm 14.5$ years. The difference in age among gender was statistically significant $(\mathrm{p}$ $=0.03$; df 199; $95 \%$ CI -9.2 to -0.4 ). Mean BMI of patients undergoing colonoscopy was $24.2 \pm 3.5$. Mean BMI of female was significantly more as compared to males ( $p=0.001 ; \mathrm{df} 199 ; 95 \%$ CI -0.48 to -2.57 ). Mean caecal intubation time was $10.7 \pm 5.4$ minutes. In males it was $10.4 \pm 5.2$ minutes while in females it was $11.2 \pm 5.6$ minutes. Difference between caecal intubation time in males and females was not significant ( $p=0.27$; df $199 ; 95 \%$ CI -2.4 to 0.7). Details are given in Table-I.

A total of four adenomas were detected by Colonoscopist-A whereas no adenomas were detected by Colonoscopist-B and C. A total of 
Table-I: Details of Age, BMI \& Caecal Time Comparison with Gender and P-values.

\begin{tabular}{lcccc}
\hline & Total Mean \pm SD & Males Mean $\pm S D$ & Female Mean $\pm S D$ & $P$ Value T-Test \\
\hline Age (years) & $36.9 \pm 15.8$ & $34.8 \pm 16.6$ & $39.6 \pm 14.5$ & 0.031 \\
BMI & $24.3 \pm 3.5$ & $23.5 \pm 3.4$ & $25.2 \pm 3.5$ & 0.001 \\
Caecal Time (minutes) & $10.7 \pm 5.4$ & $10.4 \pm 5.2$ & $11.2 \pm 5.6$ & 0.276 \\
\hline
\end{tabular}

${ }^{1}$ Significant Level $\leq 0.05$.

Table-II: Caecal Intubation Times of Colonoscopists \& Correlation with BBPS.

\begin{tabular}{lccc}
\hline Colonoscopist & CIT ${ }^{\dagger}$ (minutes) & P Value by ANNOVA & Correlation $^{\S}$ CIT $^{\ddagger}$ BBPS $^{* *}$ \\
\hline A & $9.2 \pm 4.2$ & $<0.001$ \\
B & $14.9 \pm 6.6$ & $<0.001$ & 0.041 \\
C & $10.4 \pm 5.0$ & & 0.009 \\
Mean of all 3 & $10.7 \pm 5.4$ & 0.002 \\
\hline
\end{tabular}

${ }^{\dagger}$ CIT: Caecal Intubation Time, ${ }^{\ddagger}$ Significant Level $\leq 0.05$

${ }^{\S}$ Pearson Correlation 2 Tailed, "* BBPS: Boston Bowel Preparation Scale

29 polyps were detected out of which 14 (48.3\%) polyps were detected by Colonoscopist-A, 3 $(10.3 \%)$ by Colonoscopist-B and $12(41.4 \%)$ by Colonoscopist-C.

The caecal intubation rate was $100 \%$ by all three colonoscopists. The mean CIT of Colonoscopist-A was $9.2 \pm 4.2$ minutes, Colonoscopist-B was 14.9 \pm 6.6 minutes and of Colonoscopist-C was $10.4 \pm 5.0$ minutes. Difference in caecal intubation time was significantly different among the three colonoscopists when tested with ANNOVA (P $<0.001)$. The correlation of CIT with BBPS by all three colonoscopists combined showed strong correlation with two tailed correlation at 0.002 . Individual colonoscopists caecal intubation times also showed strong correlation with BBPS scores, details are given in Table-II. No correlation of CIT was found with BMI $(p=0.479)$. Adenoma detection rates also correlated significantly with BBPS scores $(\mathrm{p}<0.001)$ but polyp detection was not found to correlate with BBPS scores $(p=0.173)$

\section{DISCUSSION}

The BBPS has been validated in a number of clinical trials., ${ }^{5,10}$ It was developed in 2009 and was designed to specify the issues affecting bowel preparation. The potential benefit of colonoscopy can only be achieved if the procedure is completed safely in minimum time with good visualization of the mucosa. Colonoscopy is widely used nowadays for therapeutic and diagnostic purposes. Its effectiveness is highly dependent on the quality of bowel preparation. Caecal intubation time is defined as the time required to reach caecum after insertion of colonoscope. Till now, the BBPS is considered the most reliable and the most relevant bowel preparation scale. It is also a simple scoring system that can be used in clinical routine practice. ${ }^{11}$ The aim of colonoscopy is to visualize the entire colon and skilled colonoscopists should intubate the caecum in at least $90 \%$ of patients. ${ }^{12}$

In our study we aimed to determine CIT \&, rate and correlate the CIT with BBPS score. Secondary objective was to see adenoma detection rate and correlation of BBPS score with BMI. The results of our study showed that increased BBPS score, i.e., good colon preparation was associated with short CIT. The result of our study corresponded with the earlier studies where mean caecal intubation time was found to be shorter with increased BBPS score. ${ }^{6}$ As consistent with the previous studies this study also highlighted the validity and reliability of BBPS. Jang JY in his study reported that poor bowel preparation increases the overall procedure time, reduces the caecal intubation rate, increases the costs of colonoscopy and increases chances of missing polyps or adenomas during colonoscopy. ${ }^{13}$ In our study adenoma detection rate was also associated with good bowel preparation but it was also linked with the skills and experience of colonoscopists.

Caecal intubation was considered successful if caecal landmarks (caecal strap fold and ileocaecal valve) were photo-documented. Bowles C et al. in their study reported $5251 / 9223$, i.e., $56.9 \%$ objectively confirmed complete colonoscopies and in $1913 / 9223(20.7 \%)$ procedures the endoscopist was unable to complete the colonoscopy. ${ }^{14}$ According to them the most common cause of this 
high failure rate was patient discomfort $(34.7 \%)$ followed by uncontrolled looping (29.7\%), poor bowel preparation $(19.6 \%)$, diverticulosis $(9.5 \%)$, adequate delineation of subtotal colitis $(2.0 \%)$, resected caecum $(7.2 \%)$ and tumor in proximal colon $(5.6 \%) .{ }^{14}$ They highlighted that one out of five incomplete colonoscopies were caused by suboptimal bowel preparation. In contrast to their result, in our study, caecal intubation rate by all three colonoscopist were $100 \%$. Although we found slight difference in mean caecal intubation time. This difference was probably due to skills and experience difference among three colonoscopists. While Akere A et al. in his study on 167 patients showed that increased BMI had negative impact on CIT but in our study we did not find any association of CIT with BMI. ${ }^{15}$

Limitation of the study: As the colonoscopies were performed by three colonoscopist so difference in their duration of experience and technique may contribute to CIT. But we try to minimize this confounder by assessing mean CIT with BBPS by all three colonoscopist separately also. Another limitation of this study was that patient's pain tolerance was not assessed prior to colonoscopy which may also contribute to the speed of colonoscopy. We used sedation and analgesia prior to every individual colonoscopy to minimize this difference in pain threshold. Another limitation of this study was that it was done in single center.

\section{CONCLUSION}

The diagnostic effectiveness of colonoscopy depends upon the quality of the preparation. This study showed that good bowel preparation improves the speed of colonoscopy, its completeness, rate of polyp and adenoma detection.

\section{REFERENCES}

1. Chung GE, Lim SH, Yang SY, Song JH, Kang HY, Kang SJ, et al. Factors that determine prolonged cecal intubation time during colonoscopy: impact of visceral adipose tissue. Scand J Gastroenterol 2014;49(10):1261-1267. doi:10.3109/00 365521.2014.950695

2. Jain D, Goyal A, Uribe J. Obesity and Cecal Intubation Time. Clin Endosc 2016;49(2):187-190. doi: 10.5946/ ce.2015.079

3. Kawasato R, Hashimoto S, Shirasawa T, Goto A, Okamoto $\mathrm{T}$, Nishikawa J, et al. Correlation between obesity and metabolic syndrome-related factors and cecal intubation time during colonoscopy. Clin Exp Gastroenterol. 2017;10:17. doi: 10.2147/CEG.S120544
4. Kim KM, Lee SH, Lee DJ, Kim KN, Seo SW, Lee HS, et al. A randomized controlled trial of comparison on time and rate of cecal and termianl Ileal intubation according to adultcolonoscope length: intermediate versus long. J Korean Med Sci. 2014;29(1):98-105. doi: 10.3346/jkms.2014.29.1.98

5. Lai EJ, Calderwood AH, Doros G, Fix OK, Jacobson BC. The Boston bowel preparation scale: a valid and reliable instrument for colonoscopy-oriented research. Gastrointest Endosc. 2009;69(3):620-625. doi: 10.1016/j.gie.2008.05.057

6. Kim EJ, Park YI, Kim YS, Park WW, Kwon SO, Park KS, et al. A Korean experience of the use of Boston bowel preparation scale: a valid and reliable instrument for colonoscopyoriented research. Saudi J Gastroenterol. 2014;20(4):219-224. doi: 10.4103/1319-3767.136950

7. Calderwood AH, Jacobson BC. Comprehensive validation of the Boston Bowel Preparation Scale. Gastrointest Endosc. 2010;72(4):686-692. doi: 10.1016/j.gie.2010.06.068

8. Gao Y, Lin JS, Zhang HD, Lin MX, Cheng CS, Wu SZ Pilot validation of the Boston Bowel Preparation Scale in China. Dig Endosc. 2013;25(2):167-173. doi:10.1111/j.14431661.2012.01356.x

9. Kluge MA, Williams JL, Wu CK, Jacobson BC, Schroy PC, 3rd, Lieberman DA, et al. Inadequate Boston Bowel Preparation Scale scores predict the risk of missed neoplasia on the next colonoscopy. Gastrointest Endosc 2018;87(3):744751. doi: $10.1016 /$ j.gie.2017.06.012

10. Parmar R MM, Rostom A, Barkun AN. Validated scales for colon cleansing: a systematic review. Am J Gastroenterol. 2016;111:197-204. doi: 10.1038/ajg.2015.417

11. Chaves Marques S. The Boston Bowel Preparation Scale: Is It Already Being Used? GE Port J Gastroenterol. 2018;25(5):219-221. doi: 10.1159/000486805

12. Moon HS, Choi EK, Seo JH, Moon JS, Song HJ, Kim KO, et al. Education and training guidelines for the board of the Korean society of gastrointestinal endoscopy. Clin Endosc. 2017;50(4):345-356. doi: 10.5946/ce.2017.106

13. Jang JY CH. Bowel preparations as quality indicators for colonoscopy. World J Gastroenterol. 2014;20(11):2746-2750. doi: 10.3748/wig.v20.i11.2746

14. Bowles C, Leicester R, Romaya C, Swarbrick E, Williams C, Epstein OJG. A prospective study of colonoscopy practice in the UK today: are we adequately prepared for national colorectal cancer screening tomorrow? Gut. 2004;53(2):277283. doi: 10.1136/gut.2003.016436

15. Akere A, Otegbayo JA. Complete colonoscopy: impact of patients' demographics and anthropometry on caecal intubation time. BMJ Open Gastroenterol. 2016;3(1):e000076. doi: 10.1136/bmjgast-2016-000076

\section{Authors' Contribution:}

HA: Did coloscopies, Study Conception.

TR: Data collection, initial manuscript writing.

MAS: Did colonoscopies, manuscript review and statistical analysis.

FSA: Manuscript writing and statistical analysis.

BFZ: Did colonoscopies, final corrections and approval of manuscript, is responsible for integrity of research.

AAS: Data collection, initial manuscript writing. 